\title{
Recent Advances in Endodontic Visualization: A Review
}

\author{
Dr. Anil Dhingra ${ }^{1}$, Dr. Nidhi Nagar ${ }^{2}$ \\ ${ }^{1}$ (Professor \& HOD, Department of Conservative Dentistry \&Endodontics, D.J. College of Dental Sciences \& \\ Research, Modinagar (U.P.), India) \\ ${ }^{2}$ (BDS, P.G. Student, Department of Conservative Dentistry \&Endodontics, D.J.College of Dental Sciences \& \\ Research ,Modinagar (U.P.), India)
}

\begin{abstract}
Advances in endodontics in the past two decades have given endodontists important new tools to save natural dentition. By providing both intense focused light as well as a high degree of magnification, the endoscope, orascope, dental loupes and operating microscopes has become an important part of the armamentarium for many endodontists. Each instrument has inherent advantages and disadvantages. Here, we describe the recent advances as well as the traditional magnification tools in field of endodontics.
\end{abstract}

Keywords: Dental loupes, Endoscope,Endodontic Visualization System,Operating microscope, Orascope.

\section{Introduction}

The art of dentistry is based on precision. The human naked eye is capable of distinguishing fine detail, but it is no match for what can be accomplished when an image is sharpened and enlarged. The microscope and other forms of magnification fill that need, especially for accomplishing endodontic procedures. (1)Enhanced magnification and illumination opened the eyes of endodontic surgeons to the intricate and complex root canal system. This advancement resulted in miniaturization of the endodontist's armamentarium. (2) Today's innovative and high-tech optical systems can deliver amazing depths-of-field and wide fields of view that enable the dentist to view a complete oral cavity in focus without having to move. (3)The use of optical magnification instruments such as endoscopes, orascopes, loupes and microscopes enables the endodontist to magnify a specified treatment field beyond that perceived by the naked eye. (4)

\section{Endoscopes}

The term endoscopy is derived from the Greek language and is literally translated as endon (within) and skopion (to see), hence the meaning, "to see within." Early endoscopists such as Hippocrates in 377 BC used primitive tube-like instruments for endoscopy. (5) With major advances in the field of medicine, a breakthrough in optical quality was achieved in 1960 by an English physician, Hopkins, who created a rod lens series that led to important advancements in the field of view, magnification, and focal length of the endoscope, resulting in a clearer image.(6)

The field of endoscopy has expanded further with the introduction of the dental endoscope. The use of rod-lens endoscope in endodontics was first reported in literature in 1979. (7) It was helpful in diagnosing dental fractures.

The traditional endoscope used in medical procedures consists of rigid glass rods and can be used in apical surgery and non-surgical endodontics. The flexible and semi-flexible endoscopes can be very valuable addition to the armamentarium. The endoscope is flexible due to special nitinol coating (Figure. 1). The optical part which is $0.9 \mathrm{~mm}$ of diameter, is a piece of equipment that enables the practitioner a magnification of up to $20 \mathrm{X}$ with clear picture with wide angle.

A $2.7 \mathrm{~mm}$ lens diameter, a $70^{\circ}$ angulation, and a $3 \mathrm{~cm}$ long rod-lens are recommended for surgical endodontic visualization and a $4 \mathrm{~mm}$ lens diameter, a $30^{\circ}$ angulation, a $4 \mathrm{~cm}$ long rod-lens are recommended for non-surgical visualization through an occlusal access opening. (Figure.2a \& 2b) (8)

The rod-lens endoscope provides clinicians greater magnification, greater clarity as compared to the microscopes and the loupes and the non-fixed field of vision. Non fixed field of vision is the ability to view treatment field at various angles and distances without losing depth of field and focus. (9)

The Modular endoscope system (Sialotechnology Ltd., Ashkelon, Israel) being based on modern technology of microendoscopes is used in small channel organs (salivary gland ductal system, tear canals) and is designed to enable the practitioner to work inside the root canal with magnification and instrument access.

The system includes three parts:

- endoscopic compact system,

- optical part that includes ocular part and the endoscope, and

- handpiece with a disposable part. (Figure. 3) (10) 


\subsection{Uses of DENTAL ENDOSCOPE}

2.1.1 Diagnosis: The dental endoscope viewing system (Dental View) is currently available as a diagnostic and therapeutic adjunct to the restorative dentist, endodontist, periodontist, oral pathologist, oral surgeon, otolaryngologist, and dental hygienist. (Figure 4)

2.1.2 Enhances Visualization: This dental endoscopic viewing system provides high magnification (24X to $50 \mathrm{X}$ ) and a light source via a fiber-optic illumination that allows to detect new carious lesion, recurrent caries, inadequate restorations in proximal boxes or class $\mathrm{V}$ restorations, intrafurcal fractures, anatomic aberrations, (eg, a palatal groove on maxillary lateral incisors), residual crown and bridge cement, oral pathologic lesions, and root fractures/perforations. (11)

2.1.3 Transillumination: In cases of tooth infraction, the endoscope can provide transillumination as a diagnostic aid. As a fiberoptic light source, it is an excellent tool for fracture detection as light may refract along fracture line. $(12,13)$

2.1.4 Apical Surgery: The surgical procedure is performed under the inspection of the endoscope with intermittent irrigation of isotonic saline and suction. The curvature of the hand-piece enables the practitioner to visualize the hidden parts of the cavity preparation, and to inspect for cracks and root fractures in the apical retrograde preparation. $(14,15)$

\subsubsection{Endoscopic Observations during Endodontic Treatment}

The endoscopic observation and treatment usually leads to detection and removal of the remaining dental pulp tissue following cleaning and shaping of the root canal walls .Lateral canals and microscopic root cracks are usually detected with high accuracy, providing better intraoperative judgment and facilitating adequate treatment. (16)

\section{Orascope}

The recently introduced flexible fiberoptic orascope is recommended for intracanal visualization, has a $.8 \mathrm{~mm}$ tip diameter, $0^{\circ}$ lens, and a working portion that is $15 \mathrm{~mm}$ in length. The term orascopy describes the use of either the rigid rod-lens endoscope or the flexible orascope in the oral cavity. (8) Orascopic endodontics is the use of orascopy for visualization in conventional and surgical endodontic treatment. (17)

The difference between an endoscope and an orascope is that:

- an orascope is made of fibre-optics (Figure.5) and

- $\quad$ an endoscope is made up of glass rods. (Figure.6)

Both an orascope and an endoscope works in conjunction with a camera, light source and a monitor. The option of a printer or a digital recorder can be added to the system for the documentation procedure.In the past, fibre optic imaging provided superior ergonomics, but suffered from poor image quality. Today a unique lens design and combined with a digital image processing system in a camera allows fibre optics to be on par with the image quality of the endoscope.

Fibre optics are made up of glass or plastic. The advantages of fibre optics in endodontics are significant. They are:

1. Small

2. Lightweight

3. Very flexible

The image quality from fibre optic usage has a direct correlation to the number of fibres and the size of the lens used in an orascope. The orascope has a 10,000 parallel visual fibres. Each visual fibre is in between 3.7 to $5 \mu$ in diameter. A ring of much larger light transmitting fibres surrounds the visual fibres for illumination of treatment field. (8)

Clinicians who use orascopic technology appreciate the fact that it has a non-fixed field of focus, which allows visualization of the treatment field at various angles and distances without losing focus and depth of field (8).

\subsection{Orascopic Visualization Technique For Conventional Endodontic Treatment}

The $0.8-\mathrm{mm}$ orascope is used to visualize within the canal system. The small fiber-optic size enables the orascope to actually go down into a canal. Prior to the placement of the 0.8 -mm fiber-optic scope, the canal must be prepared to a size No. 90 file in the coronal $15 \mathrm{~mm}$ of the canal. If the canal is not instrumented to this diameter, a wedging of the probe may occur, damaging some of the fibers within the scope. Appropriate preparation also allows the full $15 \mathrm{~mm}$ of the orascope to penetrate within the canal. If a canal is curved, the orascope may not be able to visualize around the curve because of limited flexibility. It is important to note that the canal must be dried prior to usage of the $0.8-\mathrm{mm}$ scope.The focus and depth of field of an orascope is zero 
$\mathrm{mm}$ to infinity. This allows the orascope to provide imaging of the apical third of the root without actually having to be positioned within this region of the canal. (18)

\section{Endodontic Visualization System}

The recently introduced Endodontic Visualization System (EVS) (JEDMED Instrument Company, St Louis, MO, USA) incorporates both endoscopy and orascopy into one unit. The EVS system allows for two methods of documentation. The camera head used in the EVS system is an S-video camera and, as such, documentation is usually accomplished by recording streaming video onto tape or digitized to DVD. (19)(Figure.7)

Now days the EVS II System is introduced. It also combines the fiber optic orascope and a rigid endoscope. It is said to provide optimal illumination and magnification for visualization during endodontic procedures. The system is designed to provide comfort and high quality images, and using it is said to require the same hand-eye coordination and patient positioning for ordinary procedures. The quick-connect camera handpieces can be efficiently switched to meet the needs of the procedure being performed.

\section{Dental Loupes}

Dental loupes have been the most common form of magnification used in apical surgery. Loupes are essentially two monocular microscopes with lenses mounted side by side and angled inward (convergent optics) to focus on an object. (19) Magnifying telescopes sometimes are called "loupes." A loupe can be as simple as a flat, single-element magnifier (such as plastic magnifying squares that are mounted in front of the eyes) or as complex as a multiple element telescope.Loupes are classified by the optical method in which they produce magnification.

There are three types of binocular magnifying loupes:

(1) a diopter, flat-plane, single-lens loupe,

(2) a surgical telescope with a Galileian system configuration (two lens system),

(3) a surgical telescope with a Keplarian system configuration (prism roof design that folds the path of light).(20)

5.1 Single Lens Loupe: It consists of simple magnifying lens. The only advantage of the diopter system is that is the most inexpensive system, but it is also the less desirable because the plastic lenses that uses are not always optically correct. Furthermore, the increased image size depends on being closer to the viewed object, and this can compromise posture and create stresses and abnormalities in the musculoskeletal system. (8)

5.2 Galileian Lens Loupes: The Galileian system provides a magnification range from $2 \mathrm{X}$ up to $4.5 \mathrm{X}$ and is a small, light and very compact system.

5.3 Prism Loupes: Prism loupes are the most optically advanced type of loupe magnification available today. They use refractive prisms and they are actually telescopes with complicated light paths, which provide magnifications up to 6x. Prism loupes also provide larger fields of view, wider depths of field and longer working distances than other types of loupes. (19)(Figure. 8)

\subsection{Advantages of Loupes over Microscope}

- Small in size, and easy to carry and store.

- No formal training is required and the clinician gets easily adjusted to it.Very minimal maintenance required and not as expensive as a microscope.

\subsection{Disadvantages of Loupes}

- With loupes magnification only up to $6 \mathrm{X}$ is possible.For higher magnification, microscope is required.

- Image is not stable due to head movements.

- Illumination is not as high as a microscope. Only limited magnification change is possible.

- Loupes with higher magnification are uncomfortable on the nose or head due to their large size and increased weight.

- Accessories like beam-splitter, video camera, $35 \mathrm{~mm}$ camera cannot be attached to a loupe to capture the magnified field.

\section{Operating Microscope}

Surgical Operating Microscopewas used first introduced to otolaryngology around 1950, then to neurosurgery in the 1960s, and to endodontics in the early 1990s. (21) 
Most microscopes can be configured to magnifications up to 40X and beyond but limitations in depth of field and field of view make it impractical.Clearly, if a task can be seen better it can be performed better. Fractures, POEs, and canal isthmuses can be readily seen and dealt accordingly.(19)

Recent improvements in microscope devices have been recently introduced. Modern microscopes can be easily positioned on the surgical field, and have reached a very good balancing over the complete range of movement. The surgeon can easily obtain the proper focus. (10) The microscope also serves as a useful educational tool.

\subsection{Advantages of Microscopes}

- It gives higher magnification, illumination and superior optical properties.Clinicians can easily change the working magnification.

- Using beam-splitters, which can be inserted into the optical pathway of microscope, the assistant surgeon can also view the magnified surgical field. Documentation is also possible using the beamsplitter.

- The dental operating microscopes employ Galilean optics.In Galilean optics the optical paths are parallel and focused at infinity. This reduces the need to have the eyes converge to focus and thereby reduces eye strain and fatigue.

\subsection{Disadvantages of Microscopes}

- The instrument occupies lot of space and is difficult to carry.

- Training regarding its parts and usage is a must before surgery is attempted on a patient.Alsothe surgeon's position is restricted.

- With higher magnification, the field of view and depth of focus is reduced.

- The equipment is very expensive and requires proper and regular maintenance.

\section{Conclusion}

Excellence in dentistry is both a choice and a journey, and magnification can be a powerful asset for those who seek absolute clinical accuracy.The operating microscope has revolutionized the specialty of endodontics. It represents a qualitative leap for the profession. Magnification and coaxial illumination have enormously increased the possibility of saving teeth both nonsurgically and surgically. Surgical loupes have a fixed magnification which may not allow a proper visualisation in all surgical steps.

Technological advancements in fiber-optic orascopes and rod-lens endoscopes have allowed for the development and evolution of these devices for use in clinical endodontics. The use of orascopy in conventional and surgical endodontic treatment has enabled clinicians to provide patients with improved and more predictable care.In the end, the excellent visual information can help the doctor to create more precise, more healthful, and more esthetically pleasing dentistry.

\section{References}

[1]. Donald E.Arens. Introduction to magnification in endodontics,JEsthetRestor Dent 15:426-439,2003.

[2]. Endodontics: Colleagues for Excellence AAE 2003, Advances in endodontics surgery.

[3]. Gary A .Morris, A clear view no longer means a stiff neck, Dental economics July, 1999

[4]. Ingle JI, Bakland IK. Endodontics. 6th ed. Hamilton: Bc Decker Inc; 2008.

[5]. Blakiston's New Gould Medical Dictionary. 2nd ed. New York, NY: McGraw-Hill; 1986:400.

[6]. Adamson CD, Martin DC. Endoscopic Management of Gynecologic Disease. Philadelphia, Pa: Lippincott-Raven; 1996:3-21.

[7]. Detsch S, Cunningham W, Langloss J. Endoscopy as an aid to endodontic diagnosis. J Endod 1979: 5: 60-62.

[8]. Bahcall J, Barss J. Orascopic visualization technique for conventional and surgical endodontics. IntEndod J 2003: 36 : 441-447.

[9]. Bahcall J, Barss J. Orascope vs. endoscope: a revolution in endodontic visualization. Dentistry. 2001;2:24-27.

[10]. Silvio Taschieri, Tommaso Weinstein, Igor Tsesis, Monica Bortolin, and Massimo Del Fabbro. Magnifying loupes versus surgical microscope in endodontic surgery: A four-year retrospective study. AustEndod J 2011

[11]. Greenwell H, Bissada NF, Wittwer JW. Periodontics in general practice: Perspectives on periodontal diagnosis. J Am Dent Assoc.1989;119:537-541.

[12]. Brynjulfsen A, Fristad I, Grevstad T, Hals-Kvinnsland I. Incompletely fractured teeth associated with diffuse longstanding orofacial pain: diagnosis and treatment outcome. IntEndod J 2002; 35(5): 461-466.

[13]. Filippi A, Meier ML, Lambrecht JT. Periradicular surgery with endoscopy: a clinical prospective study. SchweizMonatsschrZahnmed 2006; 116(1): 12-17.

[14]. Taschieri S, Rosano G, Weinstein T, Del Fabbro M. Endoscopic management of a lateral root lesion. A case report. Minerva Stomatol 2008; 57(11-12): 587-595.

[15]. Taschieri S, Rosano G, Francetti L, Agliardi E, Del Fabbro M. A modified technique for using the endoscope in periradicular surgery. A case report. Minerva Stomatol 2008; 57(7-8): 359-367. 
[16]. Walton R, Rivera E. Cleaning and shaping 206-238. In: Walton R, Torabinejad M, eds. Principles and practice of endodontics. 3rd ed. 2002.

[17]. Bahcall J, Barss J. Orascopy: vision for the millennium. Part II. Dent Today 1999: 18: 82-85.

[18]. James K. Bahcall, and Joseph T. Barss. Endodontic Therapy Using Orascopic Visualization. Dentistry Today, November 2003.

[19]. Richard Rubinstein. Magnification and illumination in apical surgery. Endodontic Topics 2005, 11, 56-77

[20]. Gary B. Carr and ArnaldoCastellucci. The Use of the Operating Microscope in Endodontics

[21]. Syngcuk Kim, SeunghoBaek, The microscope and endodontics, Dent Clin N Am 48 (2004) 11-18

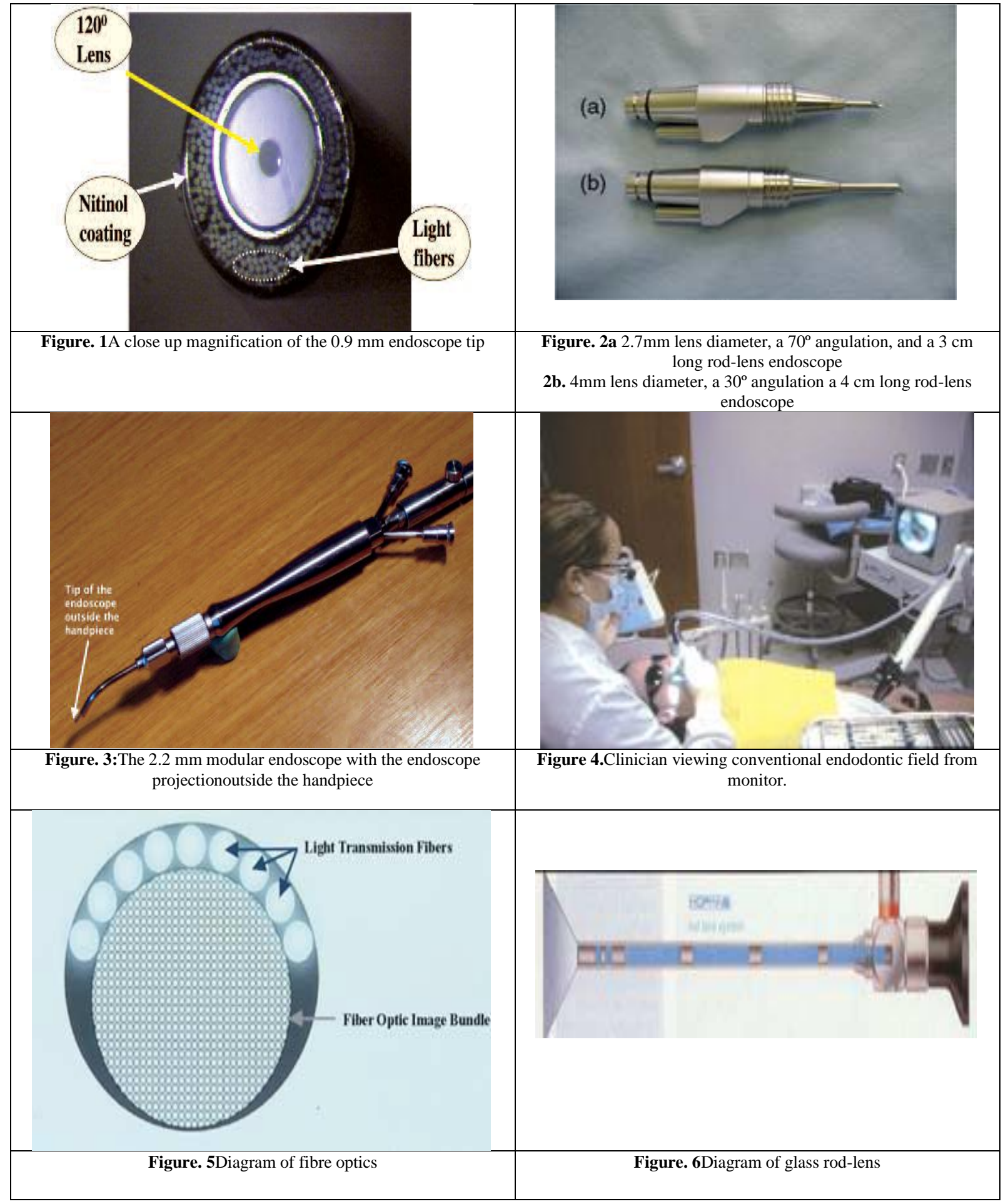




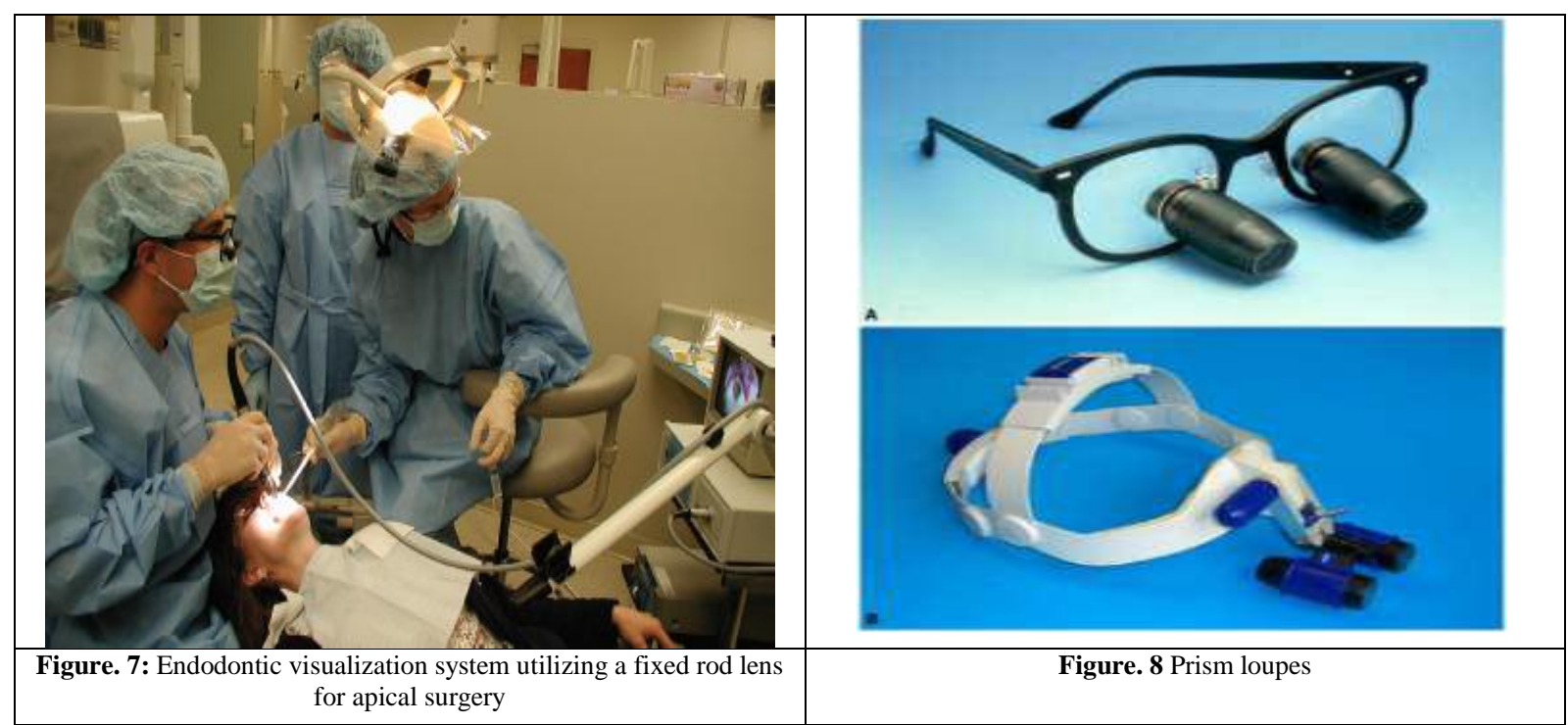

